\title{
Primary Mediastinal Malignancies in Children: Report of 22 Patients and Comparison to 197 Adults
}

\author{
Roy Temes, ${ }^{\text {a Nechol Allen, }}{ }^{\mathrm{b}}$ Theresa Chavez, ${ }^{\mathrm{c}}$ Richard Crowell, ${ }^{\mathrm{d}}$ \\ Charles Key, ${ }^{c}$ Jorge Wernly ${ }^{\mathrm{b}}$
}

${ }^{a}$ The Cleveland Clinic Foundation, Department of Cardiothoracic Surgery, Cleveland, Ohio, USA;

${ }^{b}$ The University of New Mexico Health Sciences Center, Division of Thoracic and Cardiovascular Surgery,

Albuquerque, New Mexico, USA; 'The New Mexico Tumor Registry, Albuquerque, New Mexico, USA;

${ }^{\mathrm{d}}$ The University of New Mexico Health Sciences Center, Department of Medicine, Albuquerque,

New Mexico, USA

Key Words. Mediastinal tumors · Pediatric malignancies

\begin{abstract}
Purpose. Examine a contemporary series of patients with primary pediatric malignant mediastinal tumors and determine epidemiology, histology, treatment, and survival.

Patients and Methods. All malignancies diagnosed between January 1, 1973 and December 31, 1995 were analyzed.

Results. Twenty-two patients, age 18 years or less, with pediatric primary mediastinal malignancies were identified from a database of 110,284 patients with primary malignancies. During the same period, 197 adult patients with primary mediastinal malignancies were identified. Fifty-nine percent of the pediatric patients were male. Median age was 11 years. Lymphoma was present in 55\%, neurogenic malignancies in $\mathbf{2 3 \%}$, malignant germ cell tumors in $18 \%$, and sarcoma in $5 \%$. Neurogenic tumors presented in infants and lymphomas and germ cell tumors presented in teens $(p=0.005)$. In treated children, surgery was used more often in neurogenic tumors and

germ cell tumors than in lymphomas $(p=0.002)$. Five-year survival was $74 \%$ for lymphomas, $67 \%$ for neurogenic tumors, $25 \%$ for germ cell tumors, and $61 \%$ overall $(\boldsymbol{p}=\mathbf{0 . 2 3})$. Compared to adults, children had more neurogenic tumors $(p<0.001)$ and fewer thymomas $(p=0.0499)$. There were no significant differences in staging or survival between children and adults.

Conclusions. Pediatric mediastinal malignancies occurred with a frequency of $1 / 5,013$ patients with malignant tumors. Lymphoma, neurogenic tumors, and germ cell tumors predominated. Compared to adults, children had more neurogenic tumors and fewer thymomas. Within the pediatric group, differences were found in age of presentation between histologic groups. These differences between adults and children, and between infants and teens, should be considered when evaluating a patient suspected of having mediastinal malignancy. The Oncologist 2000;5:179-184
\end{abstract}

\section{INTRODUCTION}

Primary pediatric mediastinal malignancies are rare and contemporary series reporting these tumors are uncommon. Tumor registries serve as a resource by objectively collecting the experience treating these tumors within large populations. These data allow conclusions otherwise impossible within the limited experience of individual physicians and institutions. In the future, tumor registries may become more widespread and include patients treated nationally or worldwide.

We reviewed all cases of pediatric and adult primary mediastinal malignancies diagnosed over a 23-year period to determine the histology, staging, treatment, and survival in this modern series of pediatric patients with these unusual malignancies.

\section{Materials And Methods}

The New Mexico Tumor Registry National Cancer Institute Surveillance, Epidemiology, and End Results program (SEER) statewide database was used to abstract all

Correspondence: R. Thomas Temes, M.D., Department of Surgery, Division of Thoracic and Cardiovascular Surgery, MetroHealth Medical Center, 2500 MetroHealth Drive, Cleveland, Ohio 44109-1998, USA. Telephone: 216-778-5461; Fax: 216-778-3062; e-mail: temest@ccf.org Received December 20, 1999; accepted for publication May 23, 2000. (C)AlphaMed Press 1083-7159/00/\$5.00/0 
cases of malignant primary tumors diagnosed between January 1, 1973 and December 31, 1995 within the state of New Mexico. This registry contains all malignancies diagnosed within the state and is not influenced by treatment center, treatment type, physician, or referral pattern. Although an occasional patient may be referred to New Mexico from outside the state, this is unusual and the registry represents an unbiased collection of cases within this large geographic catchment area. Cases were sought for further analysis using identifiers of origin from thymus; anterior, posterior, not otherwise specified mediastinum; intrathoracic lymph nodes; heart; and peripheral nerves and autonomic nervous system of the thorax. Primary tumors of the thyroid, parathyroid, airway, esophagus, and lung were excluded. Pediatric patients were defined as age 18 years or less at time of diagnosis. Comparisons were made to adult patients within the registry during the same time period.

Statistical analysis was performed using Fisher's Exact Test for binary variables, life table analysis for survival data, and Kruskal-Wallis analysis for age distributions.

\section{Results}

Twenty-two patients with pediatric primary mediastinal malignancies were identified from a total of 110,284 patients abstracted. Fifty-nine percent were male (13/22) and $41 \%$ were female (9/22). Histologic confirmation was made in $96 \%$ of patients (21/22). Median age was 11 years. Forty-six percent (10/22) of patients were non-Hispanic Whites, 50\% (11/22) were Hispanic Whites, and 5\% (1/22) were Black.

The histologic distribution was lymphoma in 55\% (12/22), neurogenic malignancies in $23 \%(5 / 22)$, malignant germ cell tumors in $18 \%$ (4/22), and sarcoma in 5\% (1/22) (Table 1).

Localized disease, defined as tumor confined entirely to the organ of origin, was present in $45 \%$ (10/22) of patients
(Table 2). Regional disease, defined as disease extending directly into surrounding organs or tissues and/or involved regional lymph nodes, was present in $23 \%(5 / 22)$ of patients. Distant disease, defined as tumor spread to remote areas of the body, was present in $32 \%$ (7/22) of patients. Even excluding lymphomas, tumors were localized in only 50\% $(5 / 10$ versus $10 / 22, p>0.5)$, regional in $30 \%$ (3/10 versus $5 / 22$, $p>0.5)$ and distant in $20 \%$ ( $2 / 10$ versus $7 / 22, p>0.5)$.

Five percent $(1 / 22)$ of patients received no treatment, and the remaining patients received surgical therapy, medical therapy (MED), radiation therapy (XRT), or combination therapy. MED consisted primarily of chemotherapy, but by definition within the registry, also included any hormonal or immunotherapy. Overall, MED and XRT were the most common treatment modalities and were part or all of treatment in $71 \%$ of patients (15/21) each (Table 3). Surgery was part or all of treatment in $33 \%(7 / 21)$ of the total group, and in $67 \%$ (6/9) of treated patients without lymphoproliferative malignancies $(p=0.12)$.

Lymphomas were the most common tumors and occurred in $55 \%(12 / 22)$ of patients (Table 1). Fifty-eight percent (7/12) of patients were male and $42 \%(5 / 12)$ were female. The median age was 15 years. Lymphomas were Hodgkin's disease in 58\% (7/12) and non-Hodgkin's lymphoma in $42 \%$ (5/12). All Hodgkin's lymphomas were nodular sclerosing subtypes. Tumors were staged as localized in $42 \%(5 / 12)$ of patients, regional in $17 \%(2 / 12)$, and remote in $41 \%(5 / 12)$ (Table 2). MED and XRT were part or all of treatment in $83 \%$ (10/12) of patients each (Table 3). Surgery was part or all of treatment in only $8 \%(1 / 12)$ of patients. The five-year survival for patients with lymphoma was $74 \%$ (Table 4). The five-year survival for patients with Hodgkin's lymphoma was not significantly higher than for non-Hodgkin's lymphoma (83\% versus $60 \%, p>0.5)$.

\begin{tabular}{|lllll|}
\hline \multicolumn{2}{|l}{ Table 1. Histologic classification primary mediastinal malignancies $(\%)$} & & Total & $p$ Value \\
Histology & Children & Adults & $121(55)$ & $>0.5$ \\
Lymphoma & $12(55)$ & $109(55)$ & 42 & 0.11 \\
Hodgkin's & 7 & 35 & 79 & 0.11 \\
Non-Hodgkin's & 5 & 74 & $34(16)$ & $>0.5$ \\
Germ Cell & $4(18)$ & $30(15)$ & 17 & 0.10 \\
Seminoma & 0 & 17 & 17 & 0.10 \\
Non-Seminoma & 4 & 13 & $31(14)$ & 0.0499 \\
Thymoma & $0(0)$ & $31(16)$ & $12(5)$ & $>0.5$ \\
Sarcoma & $1(5)$ & $11(6)$ & $6(3)$ & $<0.001$ \\
Neurogenic & $5(23)$ & $1(1)$ & 5 & $>0.5$ \\
Neuroblastoma & 4 & 1 & 1 & $>0.5$ \\
Ganglioneuroblastoma & 1 & 0 & $15(7)$ & NA \\
Other & $0(0)$ & $15(8)$ & 219 & NA \\
\hline Total & 22 & 197 & \\
\hline
\end{tabular}




\begin{tabular}{|c|c|c|c|c|c|c|c|c|c|}
\hline \multirow[b]{2}{*}{ Histology } & \multicolumn{3}{|c|}{ Localized } & \multicolumn{3}{|c|}{ Regional } & \multicolumn{3}{|c|}{ Remote } \\
\hline & Child & Adult & $p$ & Child & Adult & $p$ & Child & Adult & $p$ \\
\hline Lymphoma & 5 & 55 & $>0.5$ & 2 & 20 & $>0.5$ & 5 & 30 & 0.34 \\
\hline Germ Cell & 1 & 6 & $>0.5$ & 1 & 12 & $>0.5$ & 2 & 10 & $>0.5$ \\
\hline Thymoma & 0 & 5 & NA & 0 & 15 & NA & 0 & 9 & NA \\
\hline Sarcoma & 1 & 6 & $>0.5$ & 0 & 1 & $>0.5$ & 0 & 4 & $>0.5$ \\
\hline Neurogenic & 3 & 0 & $>0.5$ & 2 & 0 & $>0.5$ & 0 & 1 & 0.17 \\
\hline Total & $10(45)$ & 72 & $>0.5$ & $5(23)$ & 48 & $>0.5$ & $7(32)$ & 54 & $>0.5$ \\
\hline
\end{tabular}

\begin{tabular}{|c|c|c|c|c|c|c|c|c|c|}
\hline \multirow[b]{2}{*}{ Histology } & \multicolumn{3}{|c|}{ Surgery } & \multicolumn{3}{|c|}{ Medical } & \multicolumn{3}{|c|}{ Radiation } \\
\hline & Child & Adult & $p$ & Child & Adult & $p$ & Child & Adult & $p$ \\
\hline Lymphoma & 1 & 15 & $>0.5$ & 10 & 75 & $>0.5$ & 10 & 48 & 0.03 \\
\hline Germ Cell & 2 & 15 & $>0.5$ & 4 & 22 & $>0.5$ & 4 & 11 & 0.03 \\
\hline Thymoma & 0 & 19 & NA & 0 & 13 & NA & 0 & 21 & NA \\
\hline Sarcoma & 0 & 8 & 0.11 & 1 & 5 & $>0.5$ & 1 & 4 & $>0.5$ \\
\hline Neurogenic & 4 & 1 & $>0.5$ & 0 & 0 & $>0.5$ & 0 & 1 & 0.20 \\
\hline Total & $7(33)$ & 58 & $>0.5$ & $15(71)$ & 115 & $>0.5$ & $15(71)$ & 85 & 0.10 \\
\hline
\end{tabular}

Neurogenic tumors were the second most common pediatric primary mediastinal tumor and occurred in 23\% (5/22) of patients (Table 1$)$. Forty percent $(2 / 5)$ of patients were male and $60 \%$ (3/5) were female. Median age was less than one year and all patients were age three years or younger. Neuroblastoma was present in $80 \%$ (4/5) of patients and ganglioneuroblastoma in 20\% (1/5). Neurogenic tumors were localized in $60 \%$ (3/5) of patients, regional in $40 \%$ (2/5), and remote in none (Table 2). One patient did not receive therapy. Treated patients received surgery alone in $100 \%$ (4/4) of cases (Table 3). No patient received MED or XRT. The five-year survival of neurogenic tumors was $67 \%$ (Table 4). Only patients with localized disease survived beyond two years.

Germ cell tumors occurred in 18\% (4/22) of patients (Table 1). Seventy-five percent (3/4) of patients were male and 25\% (1/4) were female. Median age was 17 years. Germ cell tumors were malignant teratoma or teratocarcinoma in $50 \%(2 / 4)$, mixed germ cell tumor in 25\% (1/4), and embryonal carcinoma in $25 \%$ (1/4). There were no pediatric seminomas. Germ cell tumors were localized in $25 \%$ (1/4) of patients, regional in $25 \%(1 / 4)$ of patients, and remote in $50 \%(2 / 4)$ of patients (Table 2). Both MED and XRT were used in 100\% (4/4) of patients (Table 3). Surgery was part of treatment in 50\% (2/4) of patients. The five-year survival of germ cell tumors was $25 \%$ (Table 4). No patient with regional or distant disease survived beyond one year.

One patient had sarcoma (alveolar rhabdomyosarcoma). This patient was male and 11 years old at diagnosis. He had localized disease but was treated with combined MED and XRT. He survived only 18 months.

Within the pediatric group there were significant differences in age between histologies. Median age was 15 years for lymphomas, one year for neurogenic tumors, and 17 years for germ cell tumors $(p=0.005)$. There were also significant differences in treatment modality. Overall surgery was used in $100 \%(4 / 4)$ of treated patients with neurogenic tumors, $50 \%$ (2/4) of malignant germ cell tumor patients, and $8 \%(1 / 12)$ of lymphoma patients $(p=0.002)$. The five-year survival for the entire group was $61 \%$. Five-year survival was $74 \%$ for lymphomas, $67 \%$ for neurogenic tumors, and $25 \%$ for malignant germ cell tumors $(p=0.22)$. The five-year survival was not 


\begin{tabular}{|c|c|c|c|c|c|c|c|c|c|c|c|c|}
\hline \multirow[b]{2}{*}{ Histology } & \multicolumn{3}{|c|}{ Localized } & \multicolumn{3}{|c|}{ Regional } & \multicolumn{3}{|c|}{ Remote } & \multicolumn{3}{|c|}{ Overall } \\
\hline & Child & Adult & $p$ & Child & Adult & $p$ & Child & Adult & $p$ & Child & Adult & $p$ \\
\hline Lymphoma & 80 & 52 & 0.37 & 100 & 60 & $>0.5$ & 52 & 41 & $>0.5$ & 74 & 52 & 0.22 \\
\hline Hodgkin's & 100 & 81 & $>0.5$ & NA & 78 & NA & 50 & 64 & $>0.5$ & 83 & 75 & $>0.5$ \\
\hline Non-Hodgkin's & 0 & 40 & $>0.5$ & 100 & 53 & 0.49 & 50 & 27 & $>0.5$ & 60 & 41 & $>0.5$ \\
\hline Germ Cell & 100 & 24 & 0.29 & 0 & 57 & 0.46 & 0 & 58 & 0.45 & 25 & 54 & $>0.5$ \\
\hline Thymoma & $\mathrm{NA}$ & 100 & $\mathrm{NA}$ & NA & 62 & $\mathrm{NA}$ & NA & 0 & $\mathrm{NA}$ & NA & 49 & NA \\
\hline Sarcoma & 0 & 67 & 0.43 & NA & 0 & NA & NA & 0 & NA & 0 & 36 & $>0.5$ \\
\hline Neurogenic & 67 & NA & $\mathrm{NA}$ & 0 & $\mathrm{NA}$ & $\mathrm{NA}$ & NA & 0 & $\mathrm{NA}$ & 67 & 0 & $>0.5$ \\
\hline Overall & 70 & 55 & 0.50 & 80 & 58 & $>0.5$ & 37 & 34 & $>0.5$ & 61 & 51 & $>0.5$ \\
\hline
\end{tabular}

statistically different between lymphomas and non-lymphomas (74\% versus $40 \%, p=0.19$ ).

Compared to adult patients $(n=197)$ with primary mediastinal malignancies within the registry during the same time period, there were significantly more neurogenic tumors in the pediatric group $(5 / 22$ versus $1 / 197, p<0.001)$ and significantly fewer thymomas in the pediatric group $(0 / 22$ versus $31 / 197, p=0.0499)$. There were no significant differences in other histologies: sarcomas (1/22 versus $11 / 197, p>0.5)$, lymphomas and Hodgkin's disease (12/22 versus 109/197, $p>0.5$ and 7/12 versus 35/100, nine adults unknown, $p=0.13$; respectively), germ cell tumors (4/22 versus 30/197, $p>0.5$ ), and seminomas ( $0 / 4$ versus $17 / 30, p>0.5)$. There were also no significant differences in gender (males 13/22 versus 125/197, $p>0.5$ ), stage at diagnosis (localized [10/22 versus 77/187, unknown stage 10 adults, $p>0.5$ ], regional [5/22 versus $54 / 187, p>0.5]$ and remote [7/22 versus 56/187, $p>0.5]$ ), or treatment (surgery [7/21 versus 58/166, $p=0.1]$, MED [15/21 versus $115 / 166, p>0.5$ ], or XRT [15/21 versus $85 / 166$, $p=0.1])$. Pediatric patients with germ cell tumors appeared to have worse five-year survivals than adults $(25 \%$ versus $54 \%, p>0.5)$ while pediatric lymphoma and neurogenic tumor patients appeared to have better five-year survivals than adults, but none of these differences were statistically significant (74\% versus $52 \%, p=0.22$ and $67 \%$ versus $0 \%$, $p>0.5$, respectively). The overall five-year survivals were not statistically different between groups $(61 \%$ versus $51 \%$, $p>0.5)$.

\section{Comment}

Primary tumors and cysts of the mediastinum in children and adults are uncommon; they represent only 1/1,076-3,400 admissions to tertiary care medical centers, and approximately $3 \%$ of tumors within the chest [1-4]. In a collected series of primary mediastinal tumors and cysts, $25 \%-49 \%$ of these lesions are malignant [1, 2, 4-13]. Pediatric primary mediastinal tumors are even less common, but the risk of malignancy is increased to approximately $75 \%[14,15]$. We found only 22 of the 110,284 patients, or 1 of 5,013 patients with primary malignancies entered in the New Mexico tumor registry, were children with primary mediastinal malignancies.

The reported frequency of histologies depends upon the inclusion criteria of the series. Treatment and referral patterns, the presence of benign tumors and cysts, and classification by anatomic location, tissue of origin, or pathologic diagnosis will affect results. Our series encompasses all pediatric patients with malignancies diagnosed within New Mexico between 1973 and 1995. Aside from age and geographic location at time of diagnosis, there were no selection biases.

In previous reports of pediatric primary mediastinal malignancies, lymphomas were most common and present in 51\%-71\% of cases [14-18]. Our most common pediatric tumor was also lymphoma, present in $55 \%$ of patients. Hodgkin's disease has been reported to cause 33\%-56\% of pediatric mediastinal lymphomas [14-19]. Approximately 80\%-90\% are nodular sclerosing [14-18]. We found Hodgkin's disease in 58\% of pediatric lymphomas and nonHodgkin's lymphoma in 42\%. All our pediatric Hodgkin's were nodular sclerosing subtypes. Others have reported fiveyear survivals of pediatric lymphomas as $77 \%$ and survival of pediatric mediastinal Hodgkin's disease as 89\% [15]. Our pediatric lymphoma five-year survival of $74 \%$ and Hodgkin's disease five-year survival of $83 \%$ compare favorably. We found no significant differences between children and adults in frequency, staging, or survival of lymphomas or Hodgkin's disease.

Neurogenic tumors are reported to be the second most common pediatric mediastinal malignancy seen in $21 \%$ $44 \%$ of cases $[15,17,18]$. Neuroblastoma is most common and occurs in children two years of age or younger $[15,18]$. 
Ganglioneuroblastoma is the second most common [14, 15, 17]. We found malignant neurogenic tumors in $23 \%$ of children. Almost all tumors were neuroblastomas occurring in infants. Surgery was used in all treated patients. Reported survivals have been $60 \%-83 \%$ for neuroblastoma and $89 \%$ for ganglioneuroblastoma [15, 17, 18]. Our overall pediatric five-year survival was $67 \%$. Compared to adults, neurogenic mediastinal malignancies were significantly more common in children.

Mediastinal germ cell tumors arise from malignant transformation of mediastinal primordial germinal elements. Malignant germ cell tumors have been previously reported in $0 \%-4 \%$ of pediatric mediastinal malignancies $[15,20]$. Seminoma occurs in $21 \%-50 \%$ of malignant mediastinal germ cell tumors, but in children seminomas are rare [4, 5, 7-10, 13]. Most pediatric malignant mediastinal germ cell tumors are yolk sac histology [21]. We found malignant germ cell tumors in $18 \%$ of pediatric patients. The five-year survival was $25 \%$. The overall five-year survival including adults was $51 \%$, comparable to the five-year survivals of $45 \%$ for malignant germ cell tumors of all ages previously reported [3, 5, 8, 13].

Other tumors were unusual. Sarcomas have been reported in $2 \%-3 \%$ of pediatric mediastinal malignancies $[1,2,4,7,8$, $10-13,15,17,22]$. We found sarcoma in $5 \%$ of children. There were no statistical differences when compared to adults in incidence, staging, treatment, or survival. Malignant thymomas in children are rare and there were none in our pediatric series [17, 23].

Lymphomas occurred in $55 \%$ of children, neurogenic tumors in $23 \%$, germ cell tumors in $18 \%$, and sarcomas in $5 \%$. The incidence of malignant germ cell tumors, sarcomas, Hodgkin's disease, and nodular sclerosing Hodgkin's disease was higher than previously reported. Our large number of patients, the recent time interval under examination, the exclusion of benign tumors and cysts, and the absence of selection bias support our results. There were significant age differences within the pediatric group by histologic diagnosis. Neurogenic tumors occurred in infants and germ cell tumors and lymphomas presented in teenagers. Staging did not vary significantly between histologies. Surgery had a major role in the management and was used significantly more often in pediatric germ cell tumors and neurogenic tumors than in lymphomas. Five-year survival was $74 \%$ for lymphoma, $67 \%$ for neurogenic tumors, and $25 \%$ for germ cell tumors. When compared to adults, neurogenic tumors were significantly more common and thymomas significantly less common in children. Otherwise staging and survival did not differ significantly.

Since these tumors are rare, few other sizable reports exist. This study demonstrates the advantages of a large data base in studying unusual tumors. We were able to determine and compare the histology, demographics, staging, and treatment of mediastinal malignancies in children and adults and demonstrate significant differences within the pediatric population, and between children and adults. In addition to the large group of patients available for study, the database had the advantage of objectivity when compared to results derived by other means. In the future, databases such as ours may become an important means for studying and treating unusual malignant tumors.

\section{ACKNOWLEDGMenT}

We are indebted to our consultant statistician, Clifford R. Qualls, Ph.D., Professor Emeritus, Mathematics and Statistics, University of New Mexico, for invaluable assistance in analysis of data.

\section{REFERENCES}

1 Heimburger I, Battersby J, Vellios F. Primary neoplasms of the mediastinum: a fifteen year experience. Arch Surg 1963;86:120126.

2 Rubush J, Gardner I, Boyd W et al. Mediastinal tumors: review of 186 cases. J Thorac Cardiovasc Surg 1973;65:216-222.

3 Silverman N, Sabiston D Jr. Mediastinal masses. Surg Clin North Am 1980;60:757-777.

4 Wongsangiem M, Tangthangtham A. Primary tumors of the mediastinum: 190 cases analysis (1975-1995). J Med Assoc Thai 1996;79:689-697.

5 Adkins R Jr, Maples M, Hainsworth J. Primary malignant mediastinal tumors. Ann Thorac Surg 1984;38:648-659.

6 Azarow K, Pearl R, Zurcher R et al. Primary mediastinal masses: a comparison of adult and pediatric populations. J Thorac Cardiovasc Surg 1993;106:67-72.
7 Cohen A, Thompson L, Edwards F et al. Primary cysts and tumors of the mediastinum. Ann Thorac Surg 1991;51:378-386.

8 Davis R Jr, Oldham H Jr, Sabiston D Jr. Primary cysts and neoplasms of the mediastinum: recent changes in clinical presentation, methods of diagnosis, management and results. Ann Thorac Surg 1987;44:229-237.

9 Goldberg M, Burkes R. Current management of mediastinal tumors. Oncology 1994;8:99-111.

10 Hoffman O, Gillespie D, Aughenbaugh G et al. Primary mediastinal neoplasms (other than thymoma). Mayo Clin Proc 1993;68:880-891.

11 Nandi P, Wong K, Mok C et al. Primary mediastinal tumors. J R Coll Surg Edinb 1980;25:460-466.

12 Ovrum E, Birkeland S. Mediastinal tumors and cysts. Scand J Thor Cardiovasc Surg 1979;13:161-168. 
13 Rosenberg J. Neoplasms of the mediastinum. In: DeVita V Jr, Hellman S, Rosenberg S, eds. Cancer: Principles and Practice of Oncology. Philadelphia: Lippincott, 1993:759775 .

14 Grosfeld JL. Primary tumors of the chest wall and mediastinum in children. Semin Thorac Cardiovasc Surg 1994;6:235-239.

15 Grosfeld JL, Skinner MA, Rescorla FJ et al. Mediastinal tumors in children: experience with 196 cases. Ann Surg Oncol 1994;1:121-127.

16 Piira T, Perkins SL, Anderson JR et al. Primary mediastinal large cell lymphoma in children: a report from the Children's Cancer Group. Ped Pathol Lab Med 1995;15:561-570.

17 Sairanen H, Leijala M, Louhimo I. Primary mediastinal tumors in children. Eur J Cardiothorac Surg 1987;1:148151.
18 Simpson I, Campbell PE. Mediastinal masses in childhood: a review from a paediatric pathologist's point of view. Prog Ped Surg 1991;27:92-126.

19 White L, Siegel SE, Quah TC. Non-Hodgkin's lymphomas in children. I. Patterns of disease and classification. Crit Rev Oncol Hematol 1992;13:55-71.

20 Azarow KS, Pearl RH, Zurcher R et al. Primary mediastinal masses; a comparison of adult and pediatric populations. J Thorac Cardiovasc Surg 1993;106:67-72.

21 Hawkins EP. Pathology of germ cell tumors in children. Crit Rev Oncol Hematol 1990;10:165-179.

22 Conkle D, Adkins R Jr. Primary malignant tumors of the mediastinum. Ann Thorac Surg 1972;14:553-567.

23 Kaplinsky C, Mor C, Cohen IJ et al. Childhood malignant thymoma: clinical, therapeutic, and immunohistochemical considerations. Pediatr Hematol Oncol 1992;9:261-268. 